\title{
Simulation and Experiment on In-plane Carbon Nanotube Thermoelectric Generator in Parallel
}

\author{
Wenbin Huang , Xiangjun Song, Yaozhou Liu, Wanling Li, Peng Zhang, Xiaopan Liu \\ Center of Nanoscience and Technology, Institute of Mechanical Technology, Shijiazhuang, Hebei, 050003, China
}

\begin{abstract}
In order to solve the problem of larger internal resistance of thin-film thermoelectric generator (TEG) in series, which could influence the output power and restrict the application, the in-plane carbon nanotube (CNT) TEG in parallel was ingeniously researched. Utilizing the parameters of output power, conversion and energy efficiencies, the ideal and actual models of TEG in parallel were established, respectively. The thermal conduction insulating layer was taken into account, which could provide the theoretical guidance for the experimental test and engineering applications. The CNT films were prepared by the floating-catalyst chemical vapor deposition (CVD), and the experiment and properties of TEG based on CNT films were investigated. The testing circuits of conventional and gas TEGs were designed, and the output powers of the serial and parallel connecting types were tested and compared. The correctness of theoretical model and numerical analysis was proved to be valid. The novel method could effectively enhance the output power, extend the applied range of TEG in MEMS/NEMS and had a fine prospect.
\end{abstract}

\section{Introduction}

Along the quick development of micro/nano systems and their extensive applications, the power source supplied by the storage battery cannot be adapted well to the rigorous environments and requests. The size of power sources will restrict the microminiaturization and practicability of micro/nano systems, which cannot be easily replaced by the battery. The power source supplied by the storage battery cannot be adapted well to the rigorous environments and requests. The sustainable self-sufficient micro-power source, which harvests energy from the environment to power the micro/nano systems, is a feasible method of solving the supply problems [1-3]. The thermoelectric generator (TEG) is highly desirable since it can convert the heat energy into the electrical power.

TEG has many advantageous qualities over alternative devices, e.g. comparatively lightweight, completely silent, highly reliable, clean energy, no moving parts and no consumables. The heat energy could be supplied from the solar energy, automobile exhaust, production waste gas or heat dissipation of electronic components $[4,5]$. So it may play a key role for green energy production and utilization in the future. However, in order to make the TEG practical, more efficient thermoelectric materials and optimized structures must be developed and researched in recent years.

Since the discovery of CNT in 1991 [6, 7], the correlated research work has increased exponentially over the years owing to the splendid physical, chemical, structural and electrical properties $[8,9]$. It can generate electricity in the photic, thermic, magnetic or fluidic fields, which can be applied to a more complicated environment $[10,11]$. Based on the thermoelectric effects, the thermal gas and solar energy from ambient environment can be converted to electric energy.

Recently, Ref. [11, 12] reported the thermoelectric properties of CNT films and designed an integrated device to directly convert illuminating NIR light into a notable output voltage. The p-type and n-type CNT films were arranged alternatively and linked with each other in series. The output voltage can reach $0.1 \mathrm{~V}$ by setting 50 p-type CNT films and 50 n-type CNT films. However, the thin-film TEG has a larger internal resistance due to the smaller cross-sectional area. The maximum power output is directly proportional to the Seebeck coefficient and temperature difference between the hot and cold ends. But it is inversely proportional to the internal resistance. The output electrical properties are greatly influenced by the larger internal resistance. Hence, thermoelectric module (TEM) by connecting diverse p-type and n-type thermoelectric cells in parallel can reduce the internal resistance and enhance the output power. It is significant to the TEG application in diverse fields.

\section{Theoretical model}

\subsection{Ideal theoretical model}

In the ideal conditions, suppose the temperatures of the hot and cold ends remains invariable. The convection and radiation heat transfer between the hot and cold ends are 
neglected. The heat mainly transfers along the couple arms. The electrical properties effected by the electrode resistance, contact resistance and thermal resistance are neglected. When the temperature difference between the hot and cold ends is minor, The Thomson effect could be neglected. The ideal schematic diagram of the membrane thermoelectric module in parallel is shown as Fig. 1(a). Every thermoelectric cell (TEC) includes P-type and Ntype semiconductor couple arms and electrode layer. At the low temperature end, the P-type couples are connected on the substrate top surface. The N-type couples are connected on the substrate bottom surface. The membrane thermoelectric module in parallel, abbreviated 'parallel TEM' includes diverse TECs in parallel.

(a)
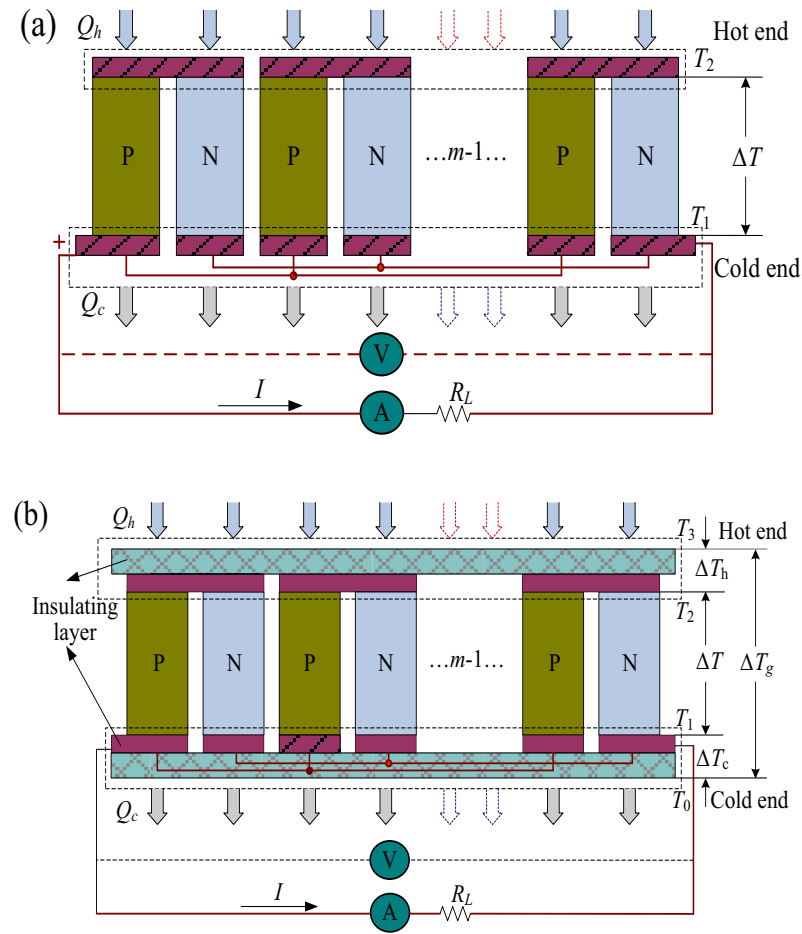

Figure 1. Ideal (a) and actual (b) schematic diagrams of membrane TEM in parallel.

Suppose that the parallel TEM is constituted by $m$ pairs of TECs. Every TEC has the same material properties and temperatures at the cold and hot ends. So the CNT couple arms have the same electrical currents $I_{i}$. The current in the main circuit is $I=m I_{i}$. The heat quantity exchanged between the heat source and hot ends of TEC is approximately equivalent.

The output power expressed by the current in the main circuit is

$$
P=Q_{h}-Q_{c}=\alpha_{p n} I \Delta T-\frac{I^{2} r}{m}
$$

The conversion efficiency is

$$
\eta=\frac{Q_{h}-Q_{c}}{Q_{h}}=\frac{m \alpha_{p n} I \Delta T-r I^{2}}{m^{2} K \Delta T+m \alpha_{p n} T_{2} I-0.5 I^{2} r}
$$

\subsection{Actual theoretical model}

When the thermal conduction insulating layer is considered in the parallel TEM, the actual schematic diagram is shown as Fig. 1(b). The positive-negative electrodes are located at the low-temperature ends. The parallel TEM requires that the P-type couple arms are connected and the N-type couple arms are connected together. So the metal wire will slightly influence the electrical properties, whether the metal wire drills through the thermal conduction insulating layer or substrate material layer. The $m$ pairs TECs in plane substrate are equivalent to the thickness of CNT films increased by $m$ times.

Suppose that the parallel TEM is constituted by $\mathrm{m}$ pairs of TECs. Every TEC has the same material properties and temperatures at the cold and hot ends. So the CNT couple arms have the same electrical currents $I_{i}$. The current in the main circuit is $I=m I_{i}$. The heat quantity exchanged between the heat source and hot ends of TEC is approximately equivalent.

The output power expressed by the current in the main circuit is

$$
\begin{aligned}
& P=\left[\alpha K_{h} K_{c} \Delta T_{g} I-r K_{h} K_{c} I^{2} / n-2 r K \bar{K} I^{2}-\alpha^{2} I^{2}\left(K_{h} T_{3}+K_{c} T_{0}\right)\right. \\
& \left.+0.5 \alpha r \Delta K_{g} I^{3} / n\right] \times\left[K_{h} K_{c}+2 n K \bar{K}-n \alpha \Delta K_{g} I-n^{2} \alpha^{2} I^{2}\right]^{-1}
\end{aligned}
$$
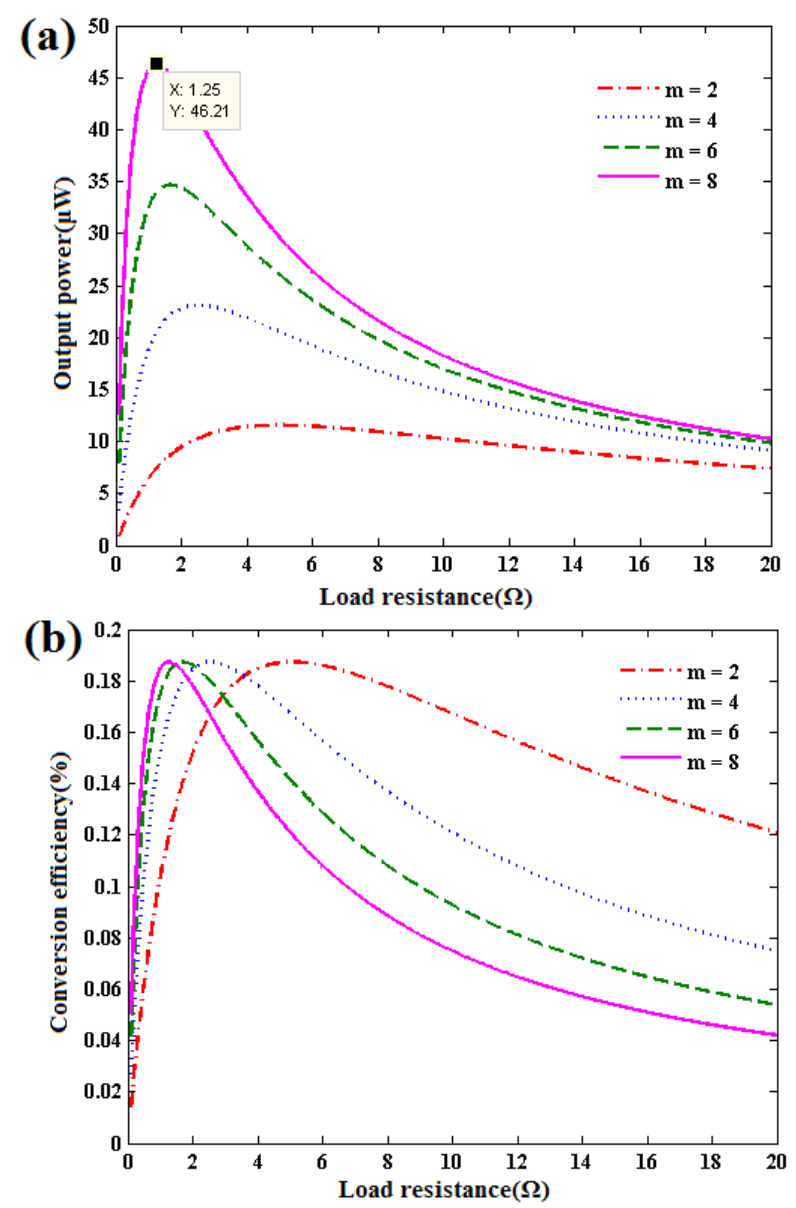

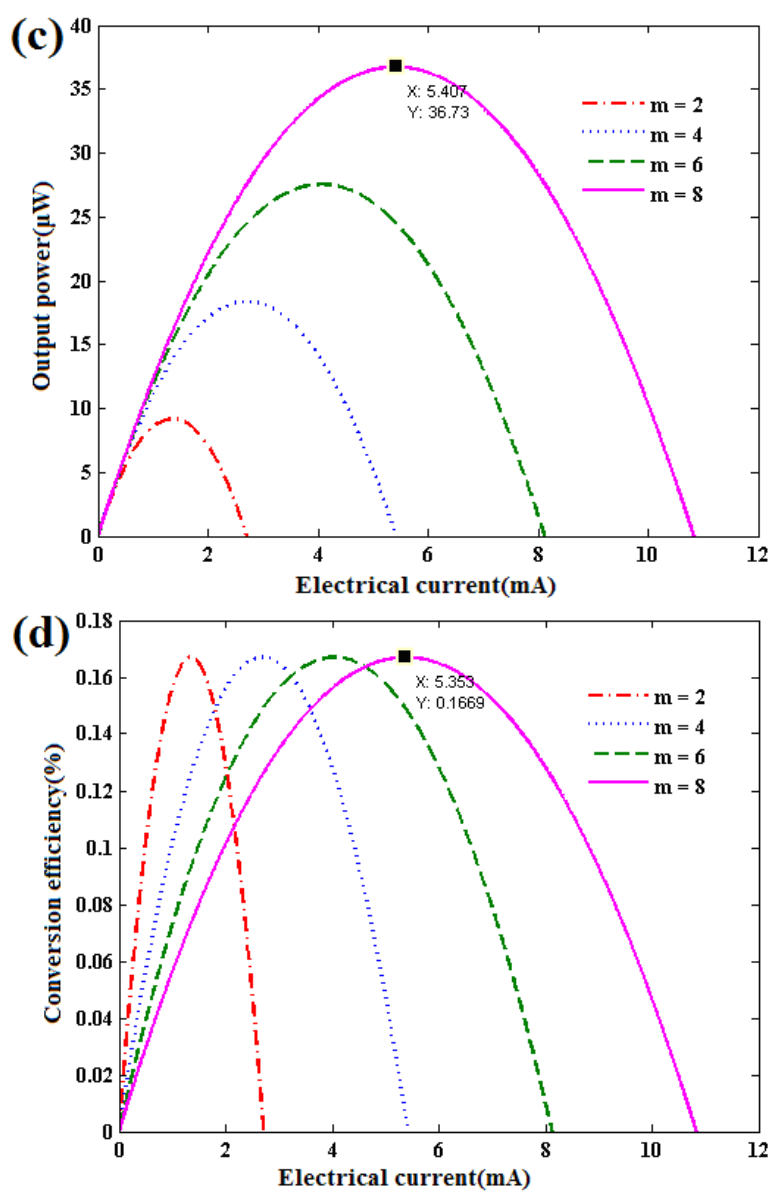

Figure 2. Performance of membrane TEM in parallel, output power (a) and conversion efficiency (b) of ideal model, output power (c) and conversion efficiency (d) of actual model.

The conversion efficiency is expressd as

$$
\begin{aligned}
& \eta_{p}=\left[\alpha K_{h} K_{c} \Delta T_{g} I-2 r K \bar{K} I^{2}+0.5 \alpha r \Delta K_{g} I^{3} / n\right] \times K_{h}^{-1} \times\left[n K K_{c} \Delta T_{g}\right. \\
& \left.+\alpha K_{c} T_{3} I-\left(0.5 r K_{c} / n+r K+\alpha^{2} T_{3}\right) I^{2}+0.5 \alpha r I^{3} / n\right]^{-1}
\end{aligned}
$$
below

The energy efficiency can be got by the equation

$$
\eta_{e}=\eta T_{3} / \Delta T_{g}
$$

When the temperature difference is smaller, the performance parameter is propitious to analyze the thermoelectric properties.

\section{Simulation and Analysis}

Assume the temperature $T_{1}=298 \mathrm{~K}, T_{2}=393 \mathrm{~K}$, the internal resistance of TEC $r=10 \Omega$, the performance analysis of membrane TEM in parallel with an ideal model is shown as Fig. 2(a) and (b). Along with the increase of parallel pair $\mathrm{m}$, the whole internal resistance of TEM obviously decreases. The output power can be enhanced by configuring the load resistances. When the internal resistance is equal to the load resistance, the output power reaches the maximal value. The maximal output power of series TEM is equal to the one of parallel TEM when they have the same connection pairs. The maximal value of conversion efficiency or exergy efficiency is independent of the parallel pair, the values are close to the ones of serial TEM.

Assuming the coefficient of thermal conductivity at the hot and cold ends are respectively $K_{\mathrm{h}}=0.8 \mathrm{~mW} / \mathrm{K}$, $K_{\mathrm{c}}=0.4 \mathrm{~mW} / \mathrm{K}$. In order to simplify the calculation, assume the surface heat transfer coefficient of TEM is fixed, $K_{\mathrm{h}}$ and $K_{\mathrm{c}}$ are directly proportional to the parallel pair. The performance analysis of membrane TEM in parallel with an actual model is shown as Fig. 2(c) and (d). The output power and the conversion efficiency could reduce due to the influence of the thermal conduction insulating layer. When $m=8$, the output power is $36.73 \mu \mathrm{W}$, the conversion efficiency is $0.1669 \%$. When the pair of TECs is equal, the output current has larger difference. So we can get the conclusion that the connecting type of TECs is determined by the load power consumption and the electrical current requested.

\section{Experiment and verification}

The in-plane TEG is composed of p-type and n-type CNT films connected by aurum electrodes. The insulator materials are filled in the gaps between p-type and n-type CNT films, which also have functions of reinforcing and smoothing the material surface. The CNT film is synthesized by a floating catalyst chemical vapor deposition (CVD) method [13, 14]. Its schematic diagram is shown in Fig. 3(a). The experimental setup is a threesection quartz tube mounted in a two-stage furnace system. The quartz tube is first flushed with the argon flow to exhaust the air. Ferrocene as catalyst precursor and sulphur as growth promoter are heated $\left(60 \sim 80{ }^{\circ} \mathrm{C}\right)$ and carried into the reactor $\left(1100{ }^{\circ} \mathrm{C}\right)$ along with the gaseous mixture of argon and methane. The large-area transparent CNT film will be synthesized in the quartz tube wall after five hours. The SEM morphology of CNT film is shown in Fig. 3(b). By doping n-type semiconductor element or coating $\operatorname{PEI}[15,16]$, the n-type CNT film can be synthesized.

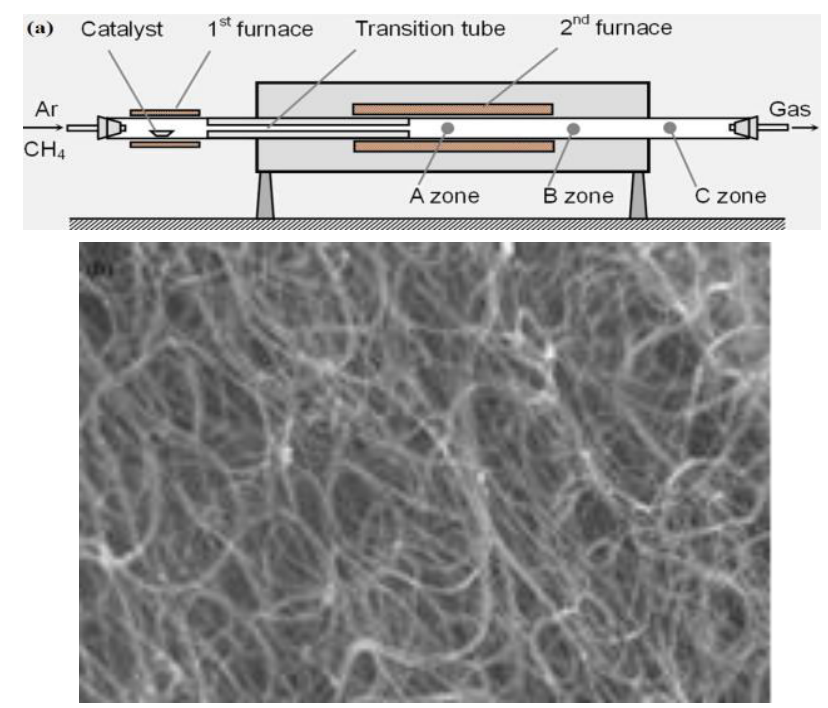

Figure 3. Schematic diagram of CVD apparatus used for synthesizing CNT membrane (a), and SEM morphology of CNT membrane (b). 

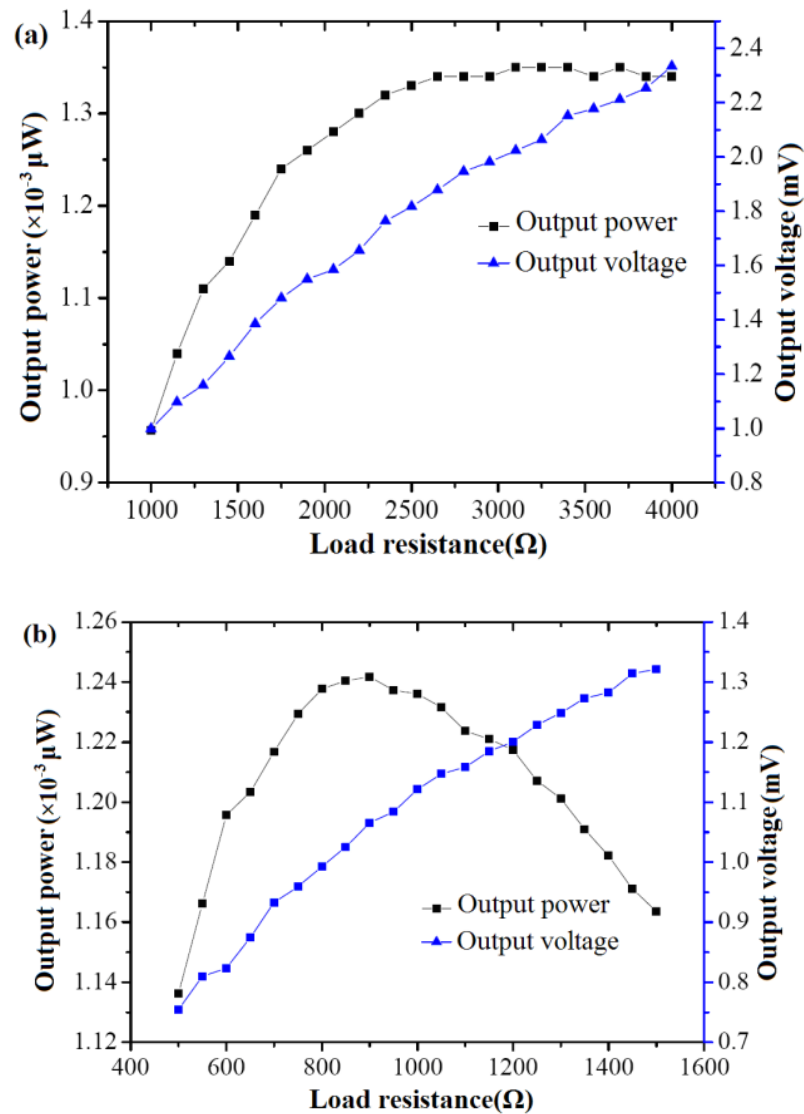

Figure 4. Comparison of thermoelectric properties between the serial (a) and parallel (b) modules.

CNT films are heated by the thermoprobe, the temperature is $348 \mathrm{~K}$ at the hot-end and $298 \mathrm{~K}$ at the cold-end. The temperature difference is $\Delta T=50 \mathrm{~K}$, which is invariable in the experiment. As shown in Fig. 4(a) and (b). The maximum output powers of the TEM in series and parallel are $1.35 \times 10^{-3} \mu \mathrm{W}$ and $1.24 \times 10^{-3} \mu \mathrm{W}$. The output power could be greatly enhanced by increasing the temperature difference and connection pairs. In the experiment, the different maximum output power is attributed to the collocation method of TEC. The parallel TEM includes two TECs are respectively disposed on the top and bottom of substrates. The larger area of electrode layer has bigger contact resistance and thermal resistance, which influence the power output. The parallel TEM can enhance the output power by reducing the internal resistance and increasing the loop current. So it is more significant in the application of thin-film TEG.

\section{Summary}

In this work, the theoretical model and optimization techniques of conventional TEG based on CNT films are researched. Utilizing the parameters of output power, conversion efficiency and energy efficiency, the ideal model and actual model of TEM in parallel are respectively established. The thermal conduction insulating layer is taken into account, which can provide the theoretical guidance for the experimental test and engineering applications. The experiment and properties of TEG based on CNT films are researched. The CNT films are prepared by CVD method. The testing circuits of conventional and gas TEG are both designed. The output voltage and power are tested in the series-parallel connecting types. The correctness of theoretical model and numerical analysis is proved to be valid. It can achieve the waste heat recovery from the solar energy, automobile exhaust, production waste gas or heat dissipation of electronic components to self-power MEMS/NEMS in the future.

\section{Acknowledgements}

The authors sincerely thank National Center for Nanoscience and Technology and Institute of Nanotechnology and Microsystems. This work was supported by National Science Foundation of China (Grant Nos. 10774032, 90921001), Key Knowledge Innovation Project of the Chinese Academy of Sciences on Water Science Research, Instrument Developing Project of the Chinese Academy of Sciences (Grant No. Y2010031).

\section{References}

1. Z.L. Wang, J.H. Song: Science, Vol. 312-315(2006), p. 242.

2. X.D. Wang, J.H. Song, J. Liu: Science, Vol. 316-319 (2007), p. 102.

3. R.S. Yang, Y. Qin, C. Li: Nano Lett., Vol. 912(2009), p. 1201.

4. D. Kraemer, P. Bed, H.P. Feng: Nat. Mater., Vol. 1014(2011), p. 532.

5. C.T. Hsu, G.Y. Huang, H.S. Chu: Appl. Energy, Vol. 88-100(2011), p. 1291.

6. S. Iijima: Nature, Vol. 354-356(1991), p. 56.

7. M.E. Itkis, F. Borondics, A.P. Yu: Science, Vol. 312315 (2006), p. 413.

8. Y.Y. Hsiao, W.C. Chang, S.L. Chen: Energy, Vol. 35-37 (2010), p. 1447.

9. Q. Cao, H.S. Kim, N. Pimparkar: Nature, Vol. $454-$ 458 (2008), p. 495

10. M.C. LeMieux, M. Roberts, S. Barman: Science, Vol. 321-325 (2008), p. 101

11. C. Yu, Y. Ryu, L. Yin: ACS Nano, Vol. 5-7 (2011), p. 1297.

12. C.H. Hu, C.H. Liu, L.Z. Chen, C.Z. Meng, S.S. Fan: ACS Nano, Vol. 4(2010), p. 4701.

13. B.C. St-Antoine, D. Menard, R. Martel: Nano Letters, Vol. 9-12 (2009), p. 3503.

14. M.A. El Khakani, V. Le Borgne, B. Aïssa: Appl. Phys. Lett., Vol. 95(2009), p. 083114-1.

15. Y.C. Zhao, L. Song and K. Deng: Adv. Mater. Vol. 20-24 (2008), p. 1772.

16. Z. Liu, K.H. Zheng, L.J. Hu: Adv. Mater., Vol. $22-$ 25 (2010), p. 999. 